\title{
$g=2$ as the natural value of the tree-level gyromagnetic ratio of elementary particles
}

\author{
Sergio Ferrara* and Massimo Porrati ${ }^{\dagger}$ \\ Theory Division, CERN, CH-1211 Geneva 23, Switzerland \\ Valentine L. Telegdi ${ }^{\ddagger}$ \\ California Institute of Technology, Pasadena, California 91125
}

(Received 7 April 1992)

\begin{abstract}
We implement at the Lagrangian level a "natural" electromagnetic coupling prescription, different from the minimal one, and proposed a long time ago by Weinberg. This prescription yields, for elementary particles of arbitrary-spin, a gyromagnetic ratio $g=2$ at the tree level. This value is already known to arise in renormalizable theories for spin $\frac{1}{2}$ and spin 1 , is suggested by the classical, relativistic equations of the spin polarization, and is also found for arbitrary-spin, charged excitations of the open string.
\end{abstract}

PACS number(s): 11.10.Ef, 11.15.Bt, 11.20.Dj

\section{INTRODUCTION}

Classically, the ratio between the total angular momentum $L$ and the magnetic moment $\mu$ of a uniformly charged rotating body is $Q / 2 M$, where $Q$ is the total charge and $M$ the mass of the body. Indeed

$$
\begin{aligned}
\boldsymbol{\mu} & =\frac{1}{2} \int d^{3} x \rho_{c} \mathbf{x} \times \mathbf{v}(\mathbf{x}) \\
& =\frac{Q}{2 M} \int d^{3} x \rho \mathbf{x} \times \mathbf{v}(\mathbf{x})=\frac{Q}{2 M} \mathbf{L} .
\end{aligned}
$$

Here $\rho$ is the constant mass density, $\rho_{c}$ is the charge density, and $\mathbf{v}(\mathbf{x})$ is the velocity of the body at the point $\mathbf{x}$. Equivalently, setting $\mu \equiv g(Q / 2 M) L$, one has $g=1$ whenever the ratio of the charge to the mass density is a constant. Thus, when spin $\frac{1}{2}$ was introduced, $g=2$ was anomalous.

Quantum mechanics and field theory change this result significantly. In the context of field theory indeed, the most natural assumption about em interactions would be that of minimal coupling according to which all derivatives of charged fields are replaced by covariant ones:

$$
\partial_{\mu} \phi \rightarrow \partial_{\mu} \phi+i e A_{\mu} \phi \equiv D_{\mu} \phi,
$$

where $e$ is the charge of the field $\phi$. This procedure is unambiguously defined only for fermionic fields, whose Lagrangian is first order in the derivatives. To define the substitution (1.2) on bosonic fields uniquely, one must first rewrite their Lagrangian in a first-order formalism [1]. This procedure gives a definite answer for the gyromagnetic ratio $g$ of elementary particles [1-5]:

$$
g=\frac{1}{s}
$$

\footnotetext{
*Also at Department of Physics, UCLA, Los Angeles, CA 90024.

tOn leave of absence from INFN, Sezione di Pisa, Pisa, Italy

†Current address: CERN, PPE Division, CH-1211 Geneva 23, Switzerland.
}

where $s$ denotes the spin of the particle.

This result, a triumph for $s=\frac{1}{2}$, is in conflict, for higher spin, with a body of (scattered) evidence that favors another answer, namely, that $g=2$ for any spin. Let us review that evidence.

(a) Up to now, the only higher-spin, charged, elementary particle that has been found in nature is the $W$ boson. At the tree level it has $g=2$, instead of $g=1$, as predicted by (1.3).

(b) The relativistic (classical) equation of motion of the polarization four-vector $S_{\mu}$ in a homogeneous external em field is [6] (the classical equation of motion used here had been anticipated by Frenkel and Thomas):

$$
\begin{aligned}
& \frac{d S^{\mu}}{d \tau}=\frac{e}{2 m} g F^{\mu v} S_{v}+\frac{e}{2 m}(g-2) U^{\mu} S_{v} F^{v \lambda} U_{\lambda}, \\
& U^{\mu}=\frac{d x^{\mu}}{d \tau}, \tau=\text { proper time } .
\end{aligned}
$$

This equation simplifies for $g=2$, irrespective of the spin of the particle.

(c) The only known example of a completely consistent theory of interacting particles with spin $>2$ is string theory [7]. For open (bosonic and supersymmetric) strings, it is possible to obtain the exact equations of motion for (massive) charged particles of arbitrary spin, moving in a constant, external em background. This has been carried out explicitly for spin 2 in Ref. [8]. The equations of motion give $g=2$ for all spins. (This point will be dealt with in greater detail below, see Sec. II.)

The aim of this paper is to provide a simple physical requirement implying $g=2$ for all "truly elementary" (pointlike) particles of any spin. ${ }^{1}$

\footnotetext{
${ }^{1}$ It is curious to notice that in (unbroken) supersymmetric electrodynamics the electron has $g=2$ even after radiative corrections, so that $g-2$ is a measure of supersymmetry-breaking effects [9].
} 
A very important condition that one imposes on physical theories is that their scattering amplitudes possess a good high-energy behavior. This requirement was advocated a long time ago by Weinberg [10]. He showed by using general properties of the $S$ matrix that this requirement implies the existence of unsubtracted dispersion relations leading to $g \approx 2$ for arbitrary-spin, weakly interacting particles. In this paper we determine in detail the Lagrangians of higher-spin massive particles interacting with the electromagnetic field, and giving rise to well-behaved high-energy scattering amplitudes; these Lagrangians are not given by minimal coupling. The Lagrangian approach presents some advantages over the $S$ matrix one: in addition to being more explicit, it also allows for the determination of all the bare electric and magnetic multipole terms.

In order to constrain Lagrangians, we ought to find an appropriate formulation of the above-mentioned conditions on scattering amplitudes. For theories of spin $\leq 1$ this formulation is known: one imposes the requirement that all tree-level amplitudes satisfy unitarity up to a center-of-mass energy $E$ much larger than the masses of all the particles involved. For particles of spin $0, \frac{1}{2}$, and 1 , this requirement has been shown to be equivalent to the statement that the most general theory is a spontaneously broken gauge theory plus an (optional) massive $\mathrm{U}(1)$ field [11].

Among the tree-level graphs having a (potentially) bad high-energy behavior, there are those involving only $W^{+}$, $W^{-}$, and photons, and describing the coupling of a $W^{+} W^{-}$pair to a high-energy photon pair (Fig. 1). Notice that these graphs depend only on the form of the em current of the W's, plus a "seagull" term ( $\gamma$ in Fig. 1), which does not affect the leading high-energy behavior of the scattering amplitude. The $W$ propagator is, in the unitary gauge,

$$
\Pi_{\mu \nu}(p)=\left[g_{\mu \nu}-\frac{p_{\mu} p_{v}}{M^{2}}\right] \frac{1}{p^{2}-M^{2}} .
$$

The term proportional to $M^{-2}$ is precisely the one responsible for the bad high-energy behavior of the graphs in Fig. 1, when the coupling of the $W^{+}, W^{-}$to photons is

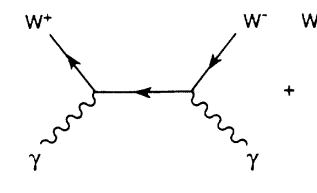

$\alpha)$

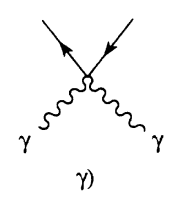

FIG. 1. Wiggly lines represent photons, straight lines $W^{+}$ and $W^{-}$. the minimal one. In fact, it is to cancel the divergences produced by the $p^{\mu} p^{v} / M^{2}$ term that one must modify the minimal em coupling of the $W$ 's by introducing the term

$$
F^{\mu v} W_{\mu}^{+} W_{v}^{-}
$$

yielding $g_{W}=2$. This term, naturally, is present in the standard model (Yang-Mills) Lagrangian, which indeed gives the following electromagnetic coupling of the $W$ s:

$L=i e F^{\mu v} W_{\mu}^{+} W_{v}^{-}+2 D_{[\mu} W_{v]}^{+} D^{[\mu} W^{-v]}+M^{2} W_{\mu}^{+} W^{-\mu}$,

$D_{[\mu} W_{v]}^{+}=\frac{1}{2} \partial_{\mu} W_{v}^{+}+i \frac{e}{2} A_{\mu} W_{v}^{+}-\frac{1}{2} \partial_{v} W_{\mu}^{+}-i \frac{e}{2} A_{v} W_{\mu}^{+}$.

The presence of a nonminimal term in gauge theories had already been noticed in Ref. [12].

The above discussion, in addition to clarifying why the standard-model value of $g_{W}$ is not the minimal one, suggests the general requirement that one should impose on any theory describing the interaction of arbitrary-spin massive particles with photons: its tree-level scattering amplitudes should not violate unitarity up to c.m. energies $E \gg M / e$. In particular, the amplitudes corresponding to the graphs of Fig. 2 must have a good high-energy behavior.

In the main body of this paper we shall show that this requirement will indeed imply that the coupling of higher-spin massive particles to the em field cannot be minimal in the usual sense, and that the nonminimal terms that must be introduced lead to $g=2$ for any spin $s$. The higher-multipole terms are thereby also constrained.

It is useful to note that our requirement can be cast in the following equivalent form: "Any theory of massive particles interacting with photons must possess a smooth $M \rightarrow 0$ fixed-charge limit."

We remark that requiring a smooth zero-mass fixedcharge limit, and thereby that tree-level amplitudes saturate the unitarity bounds only at energies $E \gg M / e$, is a necessary condition for having a sensible perturbation expansion in $e^{2}$ (in other words, a sensible electrodynamics). A theory not satisfying our requirement would indeed become strongly interacting at $E \approx M / e$.

Three supplementary remarks are in order.

(a) Supergravity allows for a spin- $\frac{3}{2}$ particle, the gravitino, interacting with Abelian gauge fields, for example, in $N=2$ models [13] or in $N=8$ models [14] broken in the manner of Scherk and Schwarz [15]. In these cases, the charge of the gravitino is proportional to the gravi-

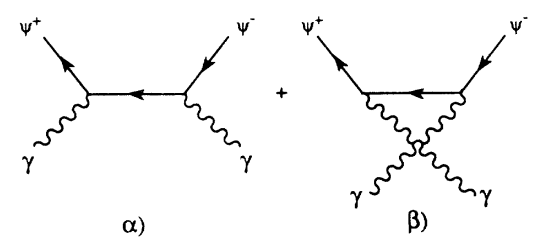

FIG. 2. $\psi$ denotes a spin- $s$ charged, massive field, represented by an oriented solid line. Contact terms are ignored because subleading at high c.m. energies. 
tino mass; therefore, the $M \rightarrow 0$ limit implies $e \rightarrow 0$. Thus, the gravitino charge cannot be identified with the electric charge: it rather describes the coupling to the "graviphoton" [13,14,15], and a good high-energy behavior of the tree-level amplitudes in Fig. 2 is compatible with a $g^{\prime}$ (proportionality constant between the total spin and the dipole magnetic moment of the graviphoton) different from 2.

(b) Interacting higher-spin Lagrangians may give rise to noncausal propagation in an external field $[16,17]$, as in the case of a massive spin- $\frac{3}{2}$ field minimally coupled to electromagnetism [16]. We shall not deal with this problem in general, since it manifests itself only at $O\left(e^{2}\right)$ and therefore can be solved only with a detailed knowledge of all (subleading) contact terms and gravitational interactions. $^{2}$ This knowledge is beyond the scope (and against the spirit) of the present paper, whose aim is rather to get results that depend as little as possible on the exact form of the higher-spin Lagrangian. We shall anyhow show in the Appendix that, at least for the case of spin $\frac{3}{2}$, appropriate nonminimal interactions (in particular those giving $g=2$ ) eliminate noncausal propagation.

(c) In general relativity it is known that a charged, rotating black hole has $g=2$ [18]. Our argument, tailored for elementary particles, cannot explain, to our knowledge, whether this result is a mere accident or is due to some deeper reason.

The present paper is organized as follows. Section II sketches the derivation of the gyromagnetic ratio in open-string theory; it is self-contained and can be skipped in a first reading, or by the reader not interested in the details of the construction. Section III deals with the consequence of imposing a smooth fixed-charge $M \rightarrow 0$ limit on massive boson fields coupled to electromagnetism, and it is shown there that $g$ must equal 2. Section IV treats the same problem for fermion fields; conclusions are given in Sec. V. The Appendix shows how nonminimal interaction terms ensure causal propagation of spin- $\frac{3}{2}$ fields in an external em background.

\section{CONSISTENT EM INTERACTIONS OF HIGHER-SPIN PARTICLES: THE OPEN-STRING EXAMPLE}

The open bosonic string propagating in a constant em background has been considered in Ref. [8], where it was proposed to use it as a means of constructing consistent interacting higher-spin equations of motion. The case of spin 2 was worked out in detail.

For our purposes, we need only to work to linear order in the external em field $F_{\mu \nu}$. To this order the bosonic string is almost identical to the free string. In particular, the Virasoro operator $L_{0}$ [7] reads, as for the free string [8],

$$
L_{0}=\sum_{m=1}^{\infty} \alpha_{-m}^{\mu} \alpha_{m \mu}+\frac{1}{2} \alpha_{-m}^{\mu} \alpha_{0 \mu} .
$$

Here $\mu=1, \ldots, D$, with $D$ equal to the dimensionality of the space which the string moves in. In open strings, charges are attached to the two end points of the string $[7,8]$. Let us call these charges $e_{0}$ and $e_{\pi}$. The charged states have a total charge $e$ equal to $e_{0}+e_{\pi}$. This charge appears in the commutation relations of the string oscillators, which read $[8]^{3}$

$$
\left[\alpha_{m}^{\mu}, \alpha_{n}^{v}\right]=\delta_{m+n, 0}\left(-m g^{\mu v}+i e F^{\mu v}\right)+O\left(F^{2}\right) .
$$

This equation tells us, in particular, that the zero modes $\alpha_{0}^{\mu}$ obey [up to terms $O\left(F^{2}\right)$ ] the commutation relations of the covariant derivatives $D^{\mu}$ defined in Eq. (1.2), and can therefore be identified with them.

The physical states of the open string are built in terms of a ground state $|0\rangle$ obeying

$$
\alpha_{m}^{\mu}|0\rangle=\partial^{\mu}|0\rangle=0, \quad \forall m>0,
$$

and a variable $x^{\mu}$, conjugate to $\partial_{\mu}$. They read

$$
\Psi=\prod_{i=1}^{N} \alpha_{-m_{i}}^{\mu_{i}} e^{i k_{\mu} x^{\mu}}|0\rangle, \quad m>0 \text {. }
$$

These states are constrained by a set of auxiliary conditions

$$
L_{n} \Psi=0, n>0,\left(L_{0}-1\right) \Psi=0,
$$

where $L_{n}$ are the Virasoro operators (see, e.g., [7]). Here we need only the equation involving $L_{0}$. Recalling that $\alpha_{0}^{\mu}=D^{\mu}$, this equation turns out to be

$$
\left(\frac{1}{2} D^{\mu} D_{\mu}+N+1\right) \Psi=0, \quad N=\sum_{m=1}^{\infty} \alpha_{-m}^{\mu} \alpha_{m \mu} .
$$

The operator $N$ satisfies the commutation relation

$$
\left[N, \alpha_{-m}^{\mu}\right]=\alpha_{-m \lambda}\left(-m g^{\lambda \mu}+i e F^{\lambda \mu}\right) .
$$

Applying this equation to (2.6), and recalling the form of the state $\Psi$ given in (2.4), one finds

$$
\left(\frac{1}{2} D^{\mu} D_{\mu}-\sum_{i=1}^{N} m_{i}+1\right) \Psi^{\mu_{1} \cdots \mu_{N}}+i e \sum_{i=1}^{N}{F_{\lambda}}^{\mu_{i}} \Psi^{\mu_{1} \cdots \mu_{i-1} \lambda \mu_{i+1} \cdots \mu_{N}}=0
$$

This equation, where we have written out explicitly the vector indices of the state $\Psi$, implies that the mass of the state $\Psi$ is $\left(2 \sum_{i=1}^{N} m_{i}-2\right)^{1 / 2}$, and that the gyromagnetic ratio $g$ is equal to 2 , as claimed.

\footnotetext{
${ }^{2}$ We recall that the gravitational background is $O\left(e^{2}\right)$ owing to Einstein's equations $R_{\mu \nu}-\frac{1}{2} g_{\mu \nu}=T_{\mu \nu}$.
}

The open superstring too can be solved in the presence of a constant background em field. This string is interesting because its spectrum does not contain tachyons (unlike the bosonic string) and it contains fermions as well.

The world-sheet action of the open superstring in a constant em field reads [7]

\footnotetext{
${ }^{3}$ Here we put the string tension $\alpha^{\prime}=\frac{1}{2}$.
} 


$$
\begin{aligned}
S=-\frac{1}{2 \pi} \int d \sigma d \tau\left\{\partial_{\alpha}\right. & X^{\mu} \partial^{\alpha} X_{\mu}-i \bar{\psi}^{\mu} \rho^{\alpha} \partial_{\alpha} \psi_{\mu} \\
+ & {\left[e_{0} \delta(\sigma)+e_{\pi} \delta(\sigma-\pi)\right] } \\
& \left.\times\left[-\frac{1}{2} F_{\mu \nu} \dot{X}^{\mu} X^{v}-\frac{1}{4} F_{\mu \nu} \bar{\psi}^{\mu} \rho^{0} \psi^{\nu}\right)\right\}
\end{aligned}
$$

Here $\sigma$ and $\tau$ are the world-sheet coordinates and $\sigma$ ranges from 0 to $\pi$; the overdot denotes derivation with respect to the time coordinate $\tau$. The $\rho^{\alpha}, \alpha=1,2$, are two-dimensional $\gamma$ matrices:

$$
\begin{aligned}
& \rho^{0}=\left(\begin{array}{cc}
0 & i \\
-i & 0
\end{array}\right), \quad \rho^{1}=\left[\begin{array}{ll}
0 & i \\
i & 0
\end{array}\right), \\
& \rho^{0}=\left(\begin{array}{cc}
-1 & 0 \\
0 & 1
\end{array}\right), \quad\left\{\rho^{\alpha}, \quad \rho^{\beta}\right\}_{+}=-2 \eta^{\alpha \beta} .
\end{aligned}
$$

The fields $\psi^{\mu}$ are two-dimensional Majorana fermions, and action (2.9) is invariant under the standard twodimensional supersymmetry transformation

$$
\delta X^{\mu}=\bar{\epsilon} \psi^{\mu}, \delta \psi^{\mu}=i \rho^{\alpha} \partial_{\alpha} x^{\mu} \epsilon .
$$

The equations of motion following from Eq. (2.11) are

$$
\partial_{\alpha} \partial^{\alpha} X^{\mu}=0, \quad \rho^{\alpha} \partial_{\alpha} \psi^{\mu}=0,
$$

supplemented by the boundary conditions

$$
\begin{array}{ll}
i\left(\psi_{-\mu}-\psi_{+\mu}\right)=-\pi e_{0} F_{\mu \nu}\left(\psi_{-}^{\nu}+\psi_{+}^{\nu}\right), & \sigma=0, \\
i\left(\psi_{-\mu} \mp \psi_{+\mu}\right)=-\pi e_{\pi} F_{\mu \nu}\left(\psi_{-}^{\nu} \pm \psi_{+}^{\nu}\right), & \sigma=\pi .
\end{array}
$$

Here $\psi_{ \pm \mu}$ are the two chiral components of $\psi_{\mu}$. In Eq. (2.14) the plus or minus signs depend on whether one chooses periodic (Ramond) or antiperiodic (NeveuSchwarz) boundary conditions for the variation of $\psi$ :

$$
\delta \psi_{+}=\delta \psi_{-}(\mathrm{R}), \delta \psi_{+}=-\delta \psi_{-} \text {(NS), } \sigma=\pi .
$$

The expansion in oscillators of the field $\psi^{\mu}$ reads

$$
\begin{aligned}
& \psi_{-}^{\mu}=\sum_{n}\left(g^{\mu v}+\pi e_{0} F^{\mu v}\right) d_{n v}, \quad \sigma=0 \\
& \psi_{+}^{\mu}=\sum_{n}\left(g^{\mu v}-\pi e_{0} F^{\mu v}\right) d_{n v}, \quad \sigma=0 .
\end{aligned}
$$

Here $n \in Z$ for Ramond boundary conditions and $n \in Z+1 / 2$ for the Neveu-Schwarz ones, while

$$
\left\{d_{n}^{\mu}, d_{m}^{v}\right\}=\delta_{n+m, 0} g^{\mu v}+O\left(F^{2}\right) .
$$

Physical states are defined in terms of a vacuum state $|0\rangle$ (NS) or $|A\rangle(\mathrm{R})$, of the operators $X^{\mu}$ and $\alpha_{m}^{\mu}$ (identical with those of the bosonic string), and of the $d_{n}^{\mu}(n, m<0)$. The NS and $R$ vacua obey

$$
\begin{aligned}
& \alpha_{m}^{\mu}|0\rangle=d_{m-1 / 2}^{\mu}|0\rangle=\partial^{\mu}|0\rangle=0, \quad m>0, \\
& \alpha_{m}^{\mu}|A\rangle=d_{m}^{\mu}|A\rangle=\partial^{\mu}|A\rangle=0, \quad m>0 .
\end{aligned}
$$

The $R$ vacuum is degenerate, and forms a spinor of the $D$-dimensional target space, of index $A$. Therefore, all NS states are $D$-dimensional bosons, and all $\mathbf{R}$ states are fermions.

The equivalent of Eq. (2.6) assumes now a different form in the NS and R sectors:

$$
\begin{aligned}
& {\left[\frac{1}{2} D^{\lambda} D_{\lambda}+\sum_{m=1}^{\infty} \alpha_{-m}^{\lambda} \alpha_{m \lambda}+\frac{1}{2}+\sum_{m=1 / 2}^{\infty}\left(-m g_{\mu \nu}+i e F_{\mu \nu}\right) d_{-m}^{\mu} d_{m}^{v}\right) \Psi^{\mu_{1} \cdots \mu_{N}}=0(\mathrm{NS}),} \\
& {\left[\frac{1}{2} D^{\lambda} D_{\lambda}+\sum_{m=1}^{\infty} \alpha_{-m}^{\lambda} \alpha_{m \lambda}+\frac{1}{2} i e F_{\mu \nu} d_{0}^{\mu} d_{0}^{v}+\sum_{m=1}^{\infty}\left(-m g_{\mu \nu}+i e F_{\mu \nu} d_{-m}^{\mu} d_{m}^{v}\right) \Psi_{A}^{\mu_{1} \cdots \mu_{N}}=0(\mathrm{R}) .\right.}
\end{aligned}
$$

The states $\Psi^{\mu_{1} \cdots \mu_{N}}$ and $\Psi_{A}^{\mu_{1} \cdots \mu_{N}}$ have the form

$$
\begin{aligned}
& \Psi^{\mu_{1} \cdots \mu_{N}}=e^{i k_{\mu} x^{\mu}} \prod_{i=1}^{N} a_{-m_{i}}^{\mu_{i}}|0\rangle, \\
& \Psi_{A}^{\mu^{1} \cdots \mu_{N}}=e^{i k_{\mu} x^{\mu}} \prod_{i=1}^{N} a_{-m_{i}}^{\mu_{i}}|A\rangle, \quad m>0 .
\end{aligned}
$$

Here $a_{m}^{\mu}$ denotes $\alpha_{m}^{\mu}$ or $d_{m}^{\mu}$ oscillators. Substituting expressions (2.20) into Eqs. (2.19), and using the commutation relations (2.17) and (2.2), we find again that, for all spins, $g=2$.

\section{III. $g=2$ FOR ALL SPINS: THE INTEGER-SPIN CASE}

String theory provided us with an example of a consistent theory describing higher-spin, charged, massive particles interacting with an em field, and in that theory we found that all charged particles have $g=2$. In this section we shall show that this result holds for all integer spins, and in any theory satisfying the general requirement stated in the Introduction, namely, that its treelevel scattering amplitudes possess a smooth zero-mass fixed-charge limit.

A method to derive free Lagrangians for massive particles of any spin was proposed by Fierz and Pauli [19]. For integer spin, explicit Lagrangians implementing that proposal were given in Ref. [1]. Those Lagrangians are written in terms of a set of fields that, for spin $s$, are symmetric-traceless tensors of rank $s, s-2, s-3, \ldots, 0$ :

$$
\begin{aligned}
& \phi_{\mu_{1} \cdots \mu_{s}}^{s}, \phi_{\mu_{1} \cdots \mu_{s-2}}^{s-2}, \phi_{\mu_{1} \cdots \mu_{s-3}}^{s-3}, \ldots, \phi^{0} ; \\
& \phi_{\mu}^{p \mu}{ }_{\mu \mu_{p}}=0 .
\end{aligned}
$$

The Lagrangian reads [1] 


$$
\begin{aligned}
& L=\phi^{* s}\left(\partial_{\mu} \partial^{\mu}-M^{2}\right) \phi^{s}+s \partial \cdot \phi^{* s} \partial \cdot \phi^{s}+\frac{s(s-1)^{2}}{2 s-1}\left[\phi^{* s-2} \partial \cdot \partial \cdot \phi^{s}-\phi^{* s-2}\left(\partial_{\mu} \partial^{\mu}-\frac{s}{s-1} M^{2}\right] \phi^{s-2}\right. \\
& \left.+\phi^{* s} \cdot \partial \cdot \partial \phi^{s-2}+\frac{(s-1)^{2}}{2 s-1} \partial \cdot \phi^{* s-2} \partial \cdot \phi^{s-2}+\cdots\right] \text {. }
\end{aligned}
$$

Here $\partial \cdot \phi^{p} \equiv \partial_{\mu} \phi^{\mu \mu_{2} \cdots \mu_{p}}$ etc. The complete system of equations of motion derived from (3.2) is

$\phi^{p}=0, p<s ;\left(\partial_{\mu} \partial^{\mu}-M^{2}\right) \phi^{s}=0, \partial \cdot \phi^{s}=0$.

In what follows, we shall not need the explicit dependence of the Lagrangian (3.2) on $\phi^{s-3}$, etc. Notice that the minimal coupling defined by Eq. (1.2) is ambiguous. For instance, integrating by parts the second term in Eq. (3.2) and then making the substitution (1.2), one finds a Lagrangian which differs from the one obtained by making substitution (1.2) directly in (3.2) by

$$
-i e s \phi^{* \mu \mu_{2} \cdots \mu_{s}} F_{\mu \nu} \phi_{\mu_{2}}^{v} \cdots \mu_{s}
$$

(From now on we suppress the index $s$ whenever no ambiguity arises.) The on-shell em current generated by the minimal substitution (1.2) into (3.2) reads

$$
J_{\mu}^{(0)}=i e \phi^{* \mu_{1} \cdots \mu_{s} \overleftrightarrow{\partial}_{\mu}} \phi_{\mu_{1} \cdots \mu_{s}} ;
$$

whereas by integration by part of (3.2) one finds

$$
\begin{aligned}
J_{\mu}^{(p)}= & i e \phi^{* \mu_{1} \cdots \mu_{s} \overleftrightarrow{\partial}_{\mu}} \phi_{\mu_{1} \cdots \mu_{s}} \\
& -i e p \partial^{v}\left(\phi_{[\mu}^{* \mu_{2} \cdots \mu_{s}} \phi_{v] \mu_{2}} \cdots \mu_{s}\right),
\end{aligned}
$$

where the integer $p$ can assume all values between 0 and $s$. In the nonrelativistic limit all fields $\phi^{s}$ with timelike indices can be neglected, and the current (3.6) gives rise to the following term in the Hamiltonian of the system:

$J_{\mu}^{(0)} A^{\mu}-i e p \phi^{* j j_{2} \cdots j_{s}} \phi^{k j_{2} \cdots j_{s}} F_{j k}, j_{a}=1,2,3$.

By noticing that, in the nonrelativistic limit,

$$
\phi^{j_{1} j_{2} \cdots j_{s}}=\frac{i}{M} \dot{\phi}^{j_{1} j_{2} \cdots j_{s}}=\frac{i}{M} \pi^{j_{1} j_{2} \cdots j_{s}},
$$

where $\pi^{j_{1} j_{2} \cdots j_{s}}$ is the canonical momentum conjugate to $\phi^{j_{1} j_{2} \cdots j_{s}}$, one discovers that the Hamiltonian contains the term

$$
J_{\mu}^{(0)} A^{\mu}+\frac{e p}{2 M s} \mathbf{S} \cdot \mathbf{B},
$$

with $B^{i}=\frac{1}{2} \epsilon^{i j k} F_{j k}$ the magnetic field, and $S^{i}$ the spin operator. Equation (3.9) says that $g=p / s$. The value $g=1 / s$ is recovered when Lagrangian (3.2) is rewritten in a first-order formalism, introducing the field strengths [1]:

$$
\begin{aligned}
H_{\mu v}{ }_{\mu \nu} \cdots \mu_{s} & =\partial_{\mu} \phi_{v}^{\mu_{2} \cdots \mu_{s}}-\frac{s-1}{s} \delta_{v}^{\left(\mu_{2}\right.} \partial \cdot \phi_{\mu}^{\left.s \mu_{3} \cdots \mu_{s}\right)} \\
& -\mu \leftrightarrow v, \text { etc. }
\end{aligned}
$$

Notice that any definition of minimal coupling gives al- ways $g \leq 1$.

If we carry out the minimal substitution on the Lagrangian (3.2), we find that the scattering amplitudes in Fig. 2 do not have a smooth zero-mass fixed-charge limit. This result comes about because the propagator of the spin-s field becomes singular in the $M \rightarrow 0$ limit. This singularity stems from the existence of null vectors of the kinetic operator of (3.2). In other words, the propagator is singular due to the existence of gauge invariances at $M=0$. To derive a result on the gyromagnetic ratio it suffices to find one of these null vectors; for instance,

$$
\begin{aligned}
& \phi_{\mu_{1} \cdots \mu_{s}}=\partial_{\left(\mu_{1}\right.} \epsilon_{\left.\mu_{2} \cdots \mu_{s}\right)}-\frac{s-1}{2 s} g_{\left(\mu_{1} \mu_{2}\right.} \partial^{\lambda} \epsilon_{\left.\lambda \mu_{3} \cdots \mu_{s}\right)}, \\
& \phi_{\mu_{1} \cdots \mu_{s-2}}=\frac{2 s-1}{s^{2}} \partial^{\lambda} \epsilon_{\lambda \mu_{1} \cdots \mu_{s-2}}, \quad \phi^{p}=0, \quad p<s-2, \\
& \partial^{\mu} \partial^{\lambda} \epsilon_{\mu \lambda u_{3}} \cdots \mu_{s} \\
& =0 .
\end{aligned}
$$

The parentheses mean total symmetrization.

Let us couple now the spin-s field to an external source $J^{s}, J^{s-2}, \ldots$, by adding to the Lagrangian (3.2) the term

$J_{\mu_{1} \cdots \mu_{s}} \phi^{\mu_{1} \cdots \mu_{s}}+J_{\mu_{1} \cdots \mu_{s-2}} \phi^{\mu_{1} \cdots \mu_{s-2}}+\cdots$.

the existence of the null vector (3.11), together with the requirement of a smooth zero-mass limit, leads to the following constraint on the source:

$$
\partial^{\lambda} J_{\lambda \mu_{1} \cdots \mu_{s-1}}+\frac{2 s-1}{s^{2}} \partial_{\left(\mu_{1}\right.} J_{\left.\mu_{2} \cdots \mu_{s-1}\right) S \mathrm{~T}}=M X_{\mu_{1} \cdots \mu_{s-1}} \text {. }
$$

The label ST means that only the symmetric traceless part of the tensor has to be kept; $X_{\mu_{1}} \cdots \mu_{s-1}$ denotes any operator with a smooth zero-mass limit.

In order to satisfy Eq. (3.13), we shall introduce additional terms in the Lagrangian, some of which proportional to Eq. (3.4). For this reason, we may choose any one of the $s+1$ possible definitions of minimal coupling: all of them will yield the same Lagrangian, when combined with the terms implied by Eq. (3.13). For sake of concreteness, we choose the definition giving rise to $J_{\mu}^{(0)}$; this generates, up to terms vanishing on shell,

$$
\begin{aligned}
J_{\mu_{1} \cdots \mu_{s}}= & i e \partial_{\lambda}\left(A^{\lambda} \phi_{\mu_{1} \cdots \mu_{s}}\right)+i e A^{\lambda} \partial_{\lambda} \phi_{\mu_{1}} \cdots \mu_{s} \\
& \left.-i e s \partial_{\left(\mu_{1}\right.}\left(A^{\lambda} \phi_{\mu_{2}} \cdots \mu_{s}\right) \lambda\right) \\
& +i e \frac{s-1}{2} g_{\left(\mu_{1} \mu_{2}\right.} \partial^{\lambda} A^{v} \phi_{\left.\mu_{3} \cdots \mu_{s}\right) \lambda v}, \\
J_{\mu_{1} \cdots \mu_{s-2}} & =i e \frac{s(s-1)^{2}}{2 s-1} \partial^{\lambda} A^{v} \phi_{\lambda \nu \mu_{1} \cdots \mu_{s-2}} .
\end{aligned}
$$


Working for convenience in the gauge $\partial_{\mu} A^{\mu}=0$, and recalling that the fields $\phi_{\mu_{1}} \cdots \mu_{s}, A_{\mu}$ are on shell (see Fig. 2), we find that the left-hand side (LHS) of Eq. (3.13) equals

$$
2 i e F^{\lambda v} \partial_{v} \phi_{\lambda \mu_{1}} \cdots \mu_{s-1}-i e M^{2} A^{\lambda} \phi_{\lambda \mu_{1}} \cdots \mu_{s-1} \text {. }
$$

The first term of Eq. (3.15) does not have the prescribed behavior for $\boldsymbol{M} \rightarrow 0$. In order to cancel it, one must add a nonminimal term to the interacting Lagrangian ${ }^{4}$ :

$$
i \alpha \phi^{* \mu \mu_{2} \cdots \mu_{s}} F_{\mu \nu} \phi_{\mu_{2} \cdots \mu_{s}}^{\nu} .
$$

This term gives the following contribution to the LHS of Eq. (3.13):

$$
i \alpha \frac{1}{s} F_{\lambda v} \partial^{\lambda} \phi_{\mu_{1} \ldots \mu_{s-1}}-i \alpha \frac{s-1}{s} \partial_{\lambda} F_{v\left(\mu_{1}\right.} \phi_{\left.\mu_{2} \ldots \mu_{s-1}\right)}^{\lambda v} .
$$

Notice that the term proportional to $F_{\mu \nu}$ cancels for

$$
\alpha=2 s e
$$

This value gives $g=2$ !

The additional term in (3.17), which vanishes for spin 1 (the $W^{ \pm}$case), and in a constant em field, can be canceled by adding the following term to the Lagrangian:

$$
\begin{aligned}
& +i \beta \phi^{* \mu \lambda \mu_{3} \cdots \mu_{s} \overleftarrow{\partial}_{\rho} F_{\lambda \sigma} \partial_{\mu} \phi^{\rho \sigma}{ }_{\mu_{3}} \cdots \mu_{s}}\left(i \delta \phi^{* \mu \lambda \mu_{3} \cdots \mu_{s}} \partial_{\rho} \partial_{\sigma} F_{\mu \nu} \partial_{\lambda} \partial^{v} \phi^{\rho \sigma}{ }_{\mu_{3} \cdots \mu_{s}}-i \beta \frac{2}{M^{2}} \phi^{* \mu_{1} \cdots \mu_{s-2}} \square\left(\partial_{\rho} F_{\sigma}{ }^{\lambda} \partial_{\lambda} \phi^{\rho \sigma}{ }_{\mu_{1}} \cdots \mu_{s-2}\right)\right. \\
& \left.+i \beta \frac{s-1}{2 s-1} \phi^{* \mu_{1} \cdots \mu_{s-2}} \partial_{\rho} F_{\sigma}{ }^{\lambda} \partial_{\lambda} \phi_{\mu_{1} \cdots \mu_{s-2}}^{\rho \sigma}+\text { H.c. }\right),
\end{aligned}
$$

where $\beta=2 s(s-1) e / M^{2}, \delta=2 s(s-1) e / M^{4}$.

The term (3.19) introduces contributions proportional to $(s-2)$ in the LHS of Eq. (3.13). These contributions can themselves be canceled by adding higher-multipole and off-diagonal terms, and so on. We do not carry through the procedure, which yields all the nonminimal multipole terms of the interacting Lagrangian, since it becomes rapidly burdensome and is not illuminating. Rather, we conclude this section by observing that the requirement of a smooth $M \rightarrow 0$ fixed-charge limit, i.e., of a good high-energy behavior, not only fixes uniquely the gyromagnetic ratio $g$ to be equal to 2 for all integer spins, but also requires the presence of ad hoc nonminimal multipole terms, which are non-power-counting renormalizable operators. In particular Eq. (3.19) implies that the ad hoc electric quadrupole moment vanishes, ${ }^{5}$ whereas nonzero magnetic octupole-moment terms must be present in the interacting theory of charged particles of spin larger than 1.

Next, we shall examine the extension of the general results obtained in this section to the case of fermionic fields.

\section{IV. $g=2$ FOR ALL SPINS: THE HALF-INTEGER-SPIN CASE}

The free Lagrangian for particles of half-integer spin $s=n+\frac{1}{2}$ can be written in terms of symmetric $\gamma$ traceless vector spinors of rank $n, n-1, \ldots, 0$ [1]:

$\psi_{\mu_{1}}^{n} \cdots \mu_{n}, \quad \psi_{\mu_{1} \cdots \mu_{n-1}}^{n-1}, \quad \psi_{\mu_{1}}^{p} \cdots \mu_{p}, \quad p<n-1, \quad i=1,2$,

\footnotetext{
${ }^{4}$ The analogous term where $F_{\mu \nu}$ is replaced by $\widetilde{F}_{\mu \nu}$ is not allowed, because it breaks parity.

${ }^{5}$ The total electric quadrupole moment is already nonzero in the spin-1 case.
}

$\gamma^{\mu} \psi_{\mu \mu_{2} \cdots \mu_{p}}^{i p}=\gamma^{\mu} \psi_{\mu \mu_{2} \cdots \mu_{n}}^{n}=\gamma^{\mu} \psi_{\mu \mu_{2} \cdots \mu_{n-1}}^{n-1}=0$.

Notice that the vector-spinors of rank lower than $n-1$ appear with multiplicity $2[1,4]$.

Here we switch to the Pauli metric $g^{\mu v}=\delta^{\mu v}$, and define the $\gamma$-matrices as

$\left\{\gamma^{\mu}, \gamma^{\nu}\right\}_{+}=\delta^{\mu \nu}, \quad \gamma^{\mu^{\dagger}}=\gamma^{\mu}, \quad \gamma^{5}=\gamma^{1} \gamma^{2} \gamma^{3} \gamma^{4}$.

The Lagrangian reads

$$
\begin{aligned}
L= & \bar{\psi}^{n}(\not-M) \psi^{n}-\frac{2 n^{2}}{2 n+1} \bar{\psi}^{n} \cdot \partial \psi^{n-1} \\
& +\frac{2 n^{2}}{2 n+1} \bar{\psi}^{n-1} \partial \cdot \psi^{n} \\
& -\frac{2 n^{2}}{2 n+1} \bar{\psi}^{n-1}\left(\not+\frac{n+1}{n} M \psi^{n-1}\right)+\cdots
\end{aligned}
$$

We shall not need the explicit dependence of (4.3) on the lower-rank vector spinors.

The minimal coupling to electromagnetism of the Lagrangian (4.3) is well defined. Substituting (1.2) into Eq. (4.3) determines the em current, which, up to terms vanishing on-shell, is

$$
J^{\mu}=i e \bar{\psi}^{\mu_{1} \cdots \mu_{n}} \gamma^{\mu} \psi_{\mu_{1} \cdots \mu_{n}}
$$

Again, we shall drop the label $n$ whenever unambiguous. The equations of motion derived from (4.3) are [1]

$$
(\not-M) \psi^{n}=0, \quad \partial \cdot \psi^{n}=0, \quad \psi^{n-1}=\psi^{i p}=0, \quad \forall i, p
$$

These equations allow us to rewrite the current $J^{\mu}$ as (see also [5]) 


$$
\begin{aligned}
J^{\mu}= & i \frac{e}{2 M} \bar{\psi}^{\mu_{1} \cdots \mu_{n} \overleftrightarrow{\partial}^{\mu}} \psi_{\mu_{1} \cdots \mu_{n}} \\
& +i \frac{e}{2 M} \partial_{v}\left(\bar{\psi}^{\mu_{1} \cdots \mu_{n}} \gamma^{\mu v} \psi_{\mu_{1} \cdots \mu_{n}}\right), \\
\gamma^{\mu \nu} \equiv & \frac{1}{2} \gamma^{\mu} \gamma^{\nu}-\frac{1}{2} \gamma_{v} \gamma^{\nu} .
\end{aligned}
$$

In the nonrelativistic limit all $\psi^{\mu_{1} \cdots \mu_{n}}$ containing at least one timelike index vanish. The nonrelativistic Hamiltonian thus contains a term

$$
\frac{e}{4 M} \bar{\psi}^{j_{1} \cdots j_{n}} \gamma^{i j} F_{i j} \psi^{j_{1} \cdots j_{n}}=\frac{e}{2 M} \frac{1}{n+\frac{1}{2}} \mathbf{B} \cdot \mathbf{S},
$$

which implies $g=1 / s$.

The reasoning used in the previous section about the $M \rightarrow 0$ limit repeats here identically. The kinetic term of the Lagrangian (4.3) possesses zero modes (gauge invariances at $M=0$ ). One of these zero modes is

$$
\begin{aligned}
& \psi_{\mu_{1} \cdots \mu_{n}}=\left.\frac{2 n(n+1)}{2 n+1} \partial_{\left(\mu_{1}\right.} \epsilon_{\left.\mu_{2} \cdots \mu_{n}\right)}\right) \\
&-\frac{n}{2 n+1} \gamma_{\left(\mu_{1}\right.} \partial \epsilon_{\left.\mu_{2} \cdots \mu_{n}\right)}, \\
& \psi_{\mu_{1} \cdots \mu_{n-1}}=\partial \epsilon_{\mu_{1} \cdots \mu_{n-1}}, \\
& \partial^{\lambda} \epsilon_{\lambda \mu_{2} \cdots \mu_{n-1}}=\gamma^{\lambda} \epsilon_{\lambda \mu_{2} \cdots \mu_{n-1}}=0 .
\end{aligned}
$$

By coupling $\psi^{n}, \psi^{n-1}$, etc., to an external source $J^{n}$, $J^{n-1}$, etc., one finds this time that the existence of a smooth zero-mass fixed-charge limit implies the equation $\partial^{\lambda} J_{\lambda_{\mu_{1}} \cdots \mu_{n-1}}+\frac{2 n+1}{2 n(n+1)} \partial J_{\mu_{1} \cdots \mu_{n-1}}=M X_{\mu_{1}} \cdots \mu_{n-1}$,

with $X_{\mu_{1}} \cdots \mu_{n-1}$ any operator having a smooth $M \rightarrow 0$ limit.

Minimal coupling gives

$\left.J_{\mu_{1} \cdots \mu_{n}}=i e A \psi_{\mu_{1}} \cdots \mu_{n}-i e \frac{n}{n+1} \gamma_{\left(\mu_{1}\right.} A^{\lambda} \psi_{\mu_{2}} \cdots \mu_{n}\right) \lambda$,

$J_{\mu_{1} \cdots \mu_{n-1}}=-i e \frac{2 n^{2}}{2 n+1} A^{\lambda} \psi_{\lambda \mu_{1}} \cdots \mu_{n-1}$.
With these values of the sources, the LHS of Eq. (4.9) becomes, up to lower-spin terms,

$-i e \gamma^{\mu} F_{\mu \nu} \psi_{\mu_{1}}^{\nu} \cdots \mu_{n-1}-i e \frac{1}{n+1} A^{\lambda} \psi_{\lambda \mu_{1}} \cdots \mu_{n-1}$.

In order to cancel the first term in the above equations, we must add to the Lagrangian a nonminimal term

$$
i \alpha \bar{\psi}^{\mu \mu_{2} \cdots \mu_{n}} F_{\mu \nu}^{+} \psi_{\mu_{2}}^{\nu} \cdots \mu_{n},
$$

where we made use of the definitions

$$
F_{\mu \nu}^{ \pm} \equiv F_{\mu \nu} \pm \frac{1}{2} \gamma_{5} \epsilon_{\mu \nu \rho \sigma} F^{\rho \sigma}, \epsilon_{1234}=+1 .
$$

Term (4.12) gives rise to the following contribution to the LHS of Eq. (4.9):

$i \alpha M \frac{1}{n} \gamma^{\mu} F_{\mu \nu} \psi_{\mu_{1}}^{\nu} \cdots \mu_{n-1}-i \alpha \frac{n-1}{n} \partial_{\rho} F_{\sigma\left(\mu_{1}\right.}^{+} \psi_{\mu_{2}}^{\rho \sigma}{ }_{\left.\mu_{n-1}\right)}$.

In deriving Eq. (4.14) we have used the identities

$$
\begin{aligned}
& \gamma^{\mu} F_{\mu \nu}^{+} \psi_{\mu_{1}}^{\nu} \cdots \mu_{n-1}=0 \text {, } \\
& F_{\mu \nu}^{+} \partial^{\mu} \psi_{\mu_{1}}^{\nu} \cdots \mu_{n-1}=M \gamma_{\mu} F^{\mu v} \psi_{\nu \mu_{1} \cdots \mu_{n-1}} .
\end{aligned}
$$

Comparing Eq. (4.14) with Eq. (4.11) we find the cancellation condition

$$
\alpha M \frac{1}{n}-e=0 \text {. }
$$

This results in $\alpha=e n / M$, which, thanks to the identity

$$
\frac{1}{2} \bar{\psi}^{\mu_{1} \cdots \mu_{n}} \gamma^{\rho \sigma} F_{\rho \sigma} \psi_{\mu_{1} \cdots \mu_{n}}=\bar{\psi}^{\mu \mu_{2} \cdots \mu_{n}} F_{\mu \nu}^{-} \psi_{\mu_{2} \cdots \mu_{n}}
$$

gives, as expected, $g=2$ for all $n$.

Notice that the additional term present in Eq. (4.14) vanishes for $n=1$, that is for spin- $\frac{3}{2}$ particles, or for constant $F_{\mu \nu}$. This term can be canceled by adding the following contributions to the action:

$i \beta \bar{\psi}^{\mu_{1} \cdots \mu_{n}} \partial_{\mu_{1}} \partial_{\rho} F_{\mu_{2} \sigma}^{+} \psi_{\mu_{3} \cdots \mu_{n}}^{\rho \sigma}+\left[i \gamma \bar{\psi}^{\mu_{1} \cdots \mu_{n-1}} \partial_{\rho} F_{\mu_{1} \sigma}^{-} \psi_{\mu_{2} \cdots \mu_{n-1}}^{\rho \sigma}+i \delta \bar{\psi}^{\mu_{1} \cdots \mu_{n-1}} \partial_{\rho} F_{\mu_{1} \sigma}^{+} \psi_{\mu_{2} \cdots \mu_{n-1}}^{\rho \sigma}+\right.$ H.c. $]$,

with

$\gamma=\frac{n\left(1-n^{2}\right)}{2 n+1} \frac{e}{M^{2}}, \quad \delta=\frac{2 n\left(1-n^{2}\right)}{(2 n+1)(n+2)} \frac{e}{M^{3}}$,

$\beta=\frac{2 n\left(1-n^{2}\right)}{n+2} \frac{e}{M^{3}}$.

The term (4.18) also gives additional contributions to the LHS of Eq. (4.9), proportional to $(n-2)$ (vanishing for spin- $\frac{5}{2}$ particles). Again, these terms can be canceled by adding higher multipole terms. We remark that, as for the integer-spin case, no ad hoc quadrupole moments are introduced, while, for generic spin, a nonminimal magnetic-octupole moment arises.

\section{CONCLUSIONS}

Our excursion into higher-spin fields has been successful: we found that a very simple and natural requirement imposed on tree-level scattering amplitudes, viz. that of a 
smooth fixed-charge $M \rightarrow 0$ limit, is powerful enough to fix the gyromagnetic ratio $g$ to its most natural value, in the Bargmann-Michel-Telegdi sense [6]. Our arguments are strictly related to those of Ref. [10], and it is reassuring to see that an explicit Lagrangian calculation such as ours, and an $S$-matrix argument, as given in [10], lead to the same conclusion about the value of $g$. We also discovered that our same requirement uniquely fixes higher-multipole terms [Eqs. (3.19), (4.18), and (4.19)], and implies the existence of quasiconserved (tensor) currents. This seems to suggest, in analogy with the spin-1 case, that a consistent theory of massive charged particles of arbitrary spin would require a large spontaneously broken local symmetry. Indeed, in the string case, there have been speculations that the infinite-slope limit would restore an infinite-dimensional symmetry [20], possibly related to a vanishing expectation value of the metric tensor $\left\langle g_{\mu \nu}\right\rangle=0$ [21]. We finally note that $g=2$ is the only value that allows for a reliable perturbative expansion, if a completely consistent theory of interacting higher-spin particles is ever to be found. A different value indeed implies that the radiative corrections, necessary in order to restore unitarity, are large; thus, whichever the value of $g$ at the tree level, it would receive huge corrections due to loops (see also Ref. [10]).

\section{ACKNOWLEDGMENTS}

We would like to thank B. Finkelstein, J. H. Schwarz, R. Stora and D. Zwanziger for useful discussions and suggestions. S.F. was supported in part by the Department of Energy of the U.S.A. under contract No. DOEAT03-88ER40384, Task E.

\section{APPENDIX: CAUSAL PROPAGATION OF SPIN- $\frac{3}{2}$ PARTICLES IN AN EXTERNAL EM FIELD}

In this appendix we show that the propagation of spin- $\frac{3}{2}$ particles in an external em field is causal, if an explicit magnetic dipole moment (Pauli term) is added to the minimal Lagrangian. Noncausal propagation [16] seems therefore to be a pathology of minimal coupling, rather than of higher-spin equations themselves.

The spin- $\frac{3}{2}$ equations of motion, in the presence of the dipole term

$$
i \alpha \bar{\psi}^{\mu} F_{\mu v}^{+} \psi^{\nu}
$$

[see Eq. (4.12)] read

$$
\begin{aligned}
\not D \psi^{\mu}-\frac{2}{3} D^{\mu} \psi-M \psi^{\mu}-\frac{1}{2} \gamma^{\mu} D_{\lambda} \psi^{\lambda} & +\frac{1}{6} \gamma^{\mu} \not D \psi \\
& +\alpha F^{+\mu \lambda} \psi_{\lambda}=0,
\end{aligned}
$$

$\not D \psi+2 M \psi-D_{\lambda} \psi^{\lambda}=0$.

In order to study the causality of propagation of waves, we must first bring the highest derivative term in a nonsingular form [16]. To this end, let us define the fields $\phi^{\mu}$, $\phi$, and $\epsilon$ by

$$
\psi^{\mu}=\phi^{\mu}+\frac{4}{3} D^{\mu} \epsilon-\frac{1}{3} \gamma^{\mu} \not D \epsilon, \quad \psi=\phi+D \epsilon, \quad D_{\mu} \phi^{\mu}=0 .
$$

The system of equations (A2) and (A3) now reads

$$
\begin{aligned}
\not D \phi^{\mu}-\frac{2}{3} D^{\mu} \phi & -\frac{4}{3} M D^{\mu} \epsilon+\frac{1}{3} M \gamma^{\mu} \not D+\frac{1}{6} \gamma^{\mu} \not D \phi \\
& +\alpha F^{+\mu \lambda} D_{\lambda \epsilon}-\frac{1}{3} \alpha F^{+\mu \lambda} \gamma_{\lambda} D \epsilon=0,
\end{aligned}
$$

$\not D \phi+2 M \phi+2 M \not \subset \epsilon=0$.

Propagation of signals in (A5) and (A6) is now studied with the method of Refs. [16,17,22]: one substitutes in (A5) and (A6) the characteristic four-vector $n_{\mu}$ to the covariant derivatives, and keeps only the leading term in $n_{\mu}$. If the resulting algebraic system admits a zero eigenvalue for some $n_{\mu}$, then the maximum speed of signal propagation is given by $n^{0} /|\mathbf{n}|[16,17,22]$. Notice that this procedure corresponds to solving Eqs. (A5) and (A6) in the eikonal approximation

$$
\begin{aligned}
& \phi^{\mu}=\hat{\phi}^{\mu} e^{i t n_{\lambda} x^{\lambda}}, \phi=\hat{\phi} e^{i t n_{\lambda} x^{\lambda}}, \\
& \epsilon=\hat{\epsilon} e^{i t n_{\lambda} x^{\lambda}}, t \rightarrow \infty,
\end{aligned}
$$

for some constant vectors $\hat{\phi}^{\mu}, \hat{\phi}$, and $\hat{\epsilon}$. Equation (A6) becomes

$$
n(\hat{\phi}+2 M \hat{\epsilon})=0 .
$$

This equation says that, for $n^{2} \neq 0, \hat{\phi}=-2 M \hat{\epsilon}$. Notice that $n^{2}=0$ is the standard light cone.

Carrying on the substitution $D_{\mu} \rightarrow$ in ${ }_{\mu}$ in Eq. (A5), and because of Eq. (A8), one finds

$$
n \widehat{\phi}^{\mu}+\frac{4}{3} \alpha F^{+\mu \lambda} n_{\lambda} \hat{\epsilon}-\frac{1}{3} \alpha F^{+\mu \lambda} \gamma_{\lambda} \hbar \hat{\epsilon}=0 .
$$

By contracting this equation with $n_{\mu}$ one arrives at

$$
\alpha F^{+\mu \lambda} n_{\mu} \gamma_{\lambda} \hat{\epsilon}=0 \text {. }
$$

If we multiply this equation by $F^{+\mu \lambda} n_{\mu} \gamma_{\lambda}$, and project over the left- and right-handed components $\hat{\epsilon}_{L}, \hat{\epsilon}_{R}$ of $\hat{\epsilon}$, we find

$$
\begin{aligned}
2 \alpha n_{\mu} n_{v}\left[\left(-\frac{1}{4} g^{\mu v} F_{\lambda \sigma} F^{\lambda \sigma}+\right.\right. & \left.F^{\mu \lambda} F_{\lambda}^{v}\right) \\
& \left.+\gamma_{\lambda \sigma} \widetilde{F}^{\mu \lambda} F^{v \sigma}\right] \hat{\epsilon}_{L}=0,
\end{aligned}
$$

and a similar term for $\hat{\epsilon}_{R}$. The term proportional to the identity matrix in Eq. (A11) is

$$
2 \alpha n_{\mu} n_{v} T^{\mu v} \text {, }
$$

where $T^{\mu \nu}$ is the stress-energy tensor of the em field.

Non-causal propagation would arise if a solution to Eq. (A10) would exist for some timelike $n_{\mu}$. By going to the Lorentz frame where $n_{\mu}=(1,0,0,0)$, this is easily seen to be impossible, thanks to Eq. (A12). In fact, $T^{00}$ vanishes only for $F_{\mu \nu}=0$, when spin- $\frac{3}{2}$ propagation is trivially causal; therefore, a timelike $n_{\mu}$ implies $\hat{\epsilon}=0$, and Eq. (A9) reduces to

$$
n \hat{\phi}^{\mu}=0 \text {. }
$$

This equation, for $n^{2} \neq 0$, admits only the trivial solution $\widehat{\phi}^{\mu}=0$.

This establishes causality of the propagation of spin- $\frac{3}{2}$ waves for all $\alpha \neq 0$. Notice that the minimal-coupling case $\alpha=0$ represents a pathological limit. 
[1] L. P. S. Singh and C. R. Hagen, Phys. Rev. D 9, 898 (1974); 9, 910 (1974).

[2] F. J. Belinfante, Phys. Rev. 92,997 (1953).

[3] K. M. Case, Phys. Rev. 94, 1442 (Z6) (1954).

[4] C. Fronsdal, Nuovo Cimento Suppl. 9, 416 (1958).

[5] J. Schwinger, Particles, Sources, and Fields (AddisonWesley, Reading, MA, 1970).

[6] V. Bargmann, L. Michel, and V. L. Telegdi, Phys. Rev. Lett. 2, 453 (1959).

[7] M. B. Green, J. H. Schwarz, and E. Witten, Superstring Theory (Cambridge University Press, Cambridge, England, 1987).

[8] P. C. Argyres and C. R. Nappi, Phys. Lett. B 224, 89 (1989).

[9] S. Ferrara and E. Remiddi, Phys. Lett. 53B, 347 (1974).

[10] S. Weinberg, in Lectures on Elementary Particles and Quantum Field Theory, Proceedings of the Summer Institute, Brandeis University, 1970, edited by S. Deser (MIT Press, Cambridge, MA, 1970), Vol. I.

[11] C. H. Llewellyn Smith, Phys. Lett. 46B, 233 (1973); J. M. Cornwall, D. N. Levin, and G. Tiktopoulos, Phys. Rev. D 10, 1145 (1974); 11, 972(E) (1975); J. M. Cornwall and G. Tiktopoulos, Phys. Rev. Lett. 35, 338 (1975).
[12] R. Finkelstein, Rev. Mod. Phys. 36, 632 (1964).

[13] S. Ferrara and P. van Nieuwenhuizen, Phys. Rev. Lett. 37, 1669 (1976).

[14] E. Cremmer and B. Julia, Nucl. Phys. B159, 141 (1979); B. de Wit and H. Nicolai, ibid. B208, 323 (1982).

[15] J. Scherk and J. H. Schwarz, Phys. Lett. 82B, 60 (1979); Nucl. Phys. B153, 61 (1979); E. Cremmer, J. Scherk, and J. H. Schwarz, Phys. Lett. 84B, 83 (1979).

[16] G. Velo and D. Zwanziger, Phys. Rev. 186, 1337 (1969); 188, 2218 (1969).

[17] G. Velo, Nucl. Phys. B43, 389 (1972).

[18] G. C. Debney, R. P. Kerr, and A. Schild, J. Math. Phys. 10, 1842 (1969).

[19] M. Fierz and W. Pauli, Proc. R. Soc. London A173, 211 (1939).

[20] D. J. Gross, Phys. Rev. Lett. 60, 1229 (1988).

[21] J. J. Atick and E. Witten, Nucl Phys. B310, 291 (1988); E. Witten, lecture given at the Meeting on String Theory of the Royal Society, London, 1988, Report No. IASSNSHEP-88/55 (unpublished).

[22] R. Courant and D. Hilbert, Methods of Mathematical Physics (Wiley-Interscience, New .York, 1962), Vol. 2, Chap. VI. 